\title{
Editorial
}

\section{Mathematical Control of Complex Systems 2013}

\author{
Zidong Wang, ${ }^{1,2}$ Hamid Reza Karimi, ${ }^{3}$ Bo Shen, ${ }^{1}$ Jun Hu, ${ }^{4,5}$ Hongli Dong, ${ }^{5,6}$ and Xiao He \\ ${ }^{1}$ School of Information Science and Technology, Donghua University, Shanghai 200051, China \\ ${ }^{2}$ Department of Information Systems and Computing, Brunel University, Uxbridge, Middlesex UB8 3PH, UK \\ ${ }^{3}$ Department of Engineering, Faculty of Engineering and Science, University of Agder, 4898 Grimstad, Norway \\ ${ }^{4}$ Department of Applied Mathematics, Harbin University of Science and Technology, Harbin 150080, China \\ ${ }^{5}$ Research Institute of Intelligent Control and Systems, Harbin Institute of Technology, Harbin 150001, China \\ ${ }^{6}$ College of Electrical and Information Engineering, Northeast Petroleum University, Daqing 163318, China \\ ${ }^{7}$ Department of Automation, Tsinghua University, Beijing 100084, China
}

Correspondence should be addressed to Zidong Wang; zidong.wang@brunel.ac.uk

Received 1 December 2013; Accepted 1 December 2013; Published 23 January 2014

Copyright (C) 2014 Zidong Wang et al. This is an open access article distributed under the Creative Commons Attribution License, which permits unrestricted use, distribution, and reproduction in any medium, provided the original work is properly cited.

Mathematical control of complex systems have already become an ideal research area for control engineers, mathematicians, computer scientists, and biologists to understand, manage, analyze, and interpret functional information/ dynamical behaviours from real-world complex dynamical systems, such as communication systems, process control, environmental systems, intelligent manufacturing systems, transportation systems, and structural systems. This special issue aims to bring together the latest/innovative knowledge and advances in mathematics for handling complex systems. Topics include, but are not limited to the following: control systems theory (behavioural systems, networked control systems, delay systems, distributed systems, infinite-dimensional systems, and positive systems); networked control (channel capacity constraints, control over communication networks, distributed filtering and control, information theory and control, and sensor networks); and stochastic systems (nonlinear filtering, nonparametric methods, particle filtering, partial identification, stochastic control, stochastic realization, system identification).

We have solicited submissions to this special issue from control engineers, electrical engineers, computer scientists, and mathematicians. After a rigorous peer review process, 31 papers have been selected that provide overviews, solutions, or early promises, to manage, analyze, and interpret dynamical behaviours of complex systems. These papers have covered both the theoretical and practical aspects of complex systems in the broad areas of dynamical systems, mathematics, statistics, operational research, and practical engineering.

This special issue starts with two survey papers on the recent advances of recursive filtering (sliding mode design) for networked nonlinear stochastic systems and distributed filtering (fault detection) for sensor networks. Specifically, in the paper entitled "Recent advances on recursive filtering and sliding mode design for networked nonlinear stochastic systems: a survey" by $\mathrm{J}$. $\mathrm{Hu}$ et al., the focus is to provide a timely review on the recent advances of the recursive filtering and sliding mode design for nonlinear stochastic systems with network-induced phenomena. The network-induced phenomena under consideration include missing measurements, fading measurements, signal quantization, probabilistic sensor delays, sensor saturations, and randomly occurring nonlinearities. The recent developments of the networkinduced phenomena are first summarized. Various filtering and sliding mode design for nonlinear stochastic systems are reviewed in great detail and some interesting yet challenging issues are raised. Latest results on recursive filtering and sliding mode designs for discrete-time nonlinear stochastic systems with network-induced phenomena are reviewed. Subsequently, a bibliographical review is provided in " $A$ survey on distributed filtering and fault detection for sensor networks" by $\mathrm{H}$. Dong et al. on distributed filtering and fault detection problems over sensor networks. The algorithms employed to study the distributed filtering and detection 
problems are categorized and then discussed. In addition, some recent advances in distributed detection problems for faulty sensors and fault-events are also summarized in great detail. Finally, some concluding remarks are drawn and the future research challenges for distributed filtering and fault detection for sensor networks are pointed out.

During the past decades, the problems of performance analysis of complex systems have received significant research attention. In the paper entitled "Numerical optimization design of dynamic quantizer via matrix uncertainty approach" by K. Sawada and S. Shin, a numerical optimization method is proposed for the continuous-time dynamic quantizer under switching speed and quantized accuracy constraints. The design problem is discussed via sampled-data control framework and the case of temporal and spatial resolution constraints can be addressed in analysis and synthesis, simultaneously. Also, a new insight is presented for the two-step design of the existing continuous-time optimal quantizer. The performance monitoring and reliability analysis is discussed in "Performance reliability prediction of complex system based on the condition monitoring information" by $\mathrm{H}$. Wang and Y. Jiang. Aircraft engine performance degradation process is described by using the Wiener process to forecast aeroengine performance reliability taking the condition monitoring information into account. The proposed method integrates the performance monitoring and reliability analysis into one framework by making full use of a variety of condition monitoring information. In the work entitled "The method of Lyapunov function and exponential stability of impulsive delay systems with delayed impulses" by P. Cheng et al., the problem of exponential stability is studied for a class of general impulsive delay systems with delayed impulses. By using the Lyapunov function method, some Lyapunov-based sufficient conditions are derived to ensure the exponential stability. Their applications to linear impulsive systems with time-varying delays are also proposed, and a set of sufficient conditions for exponential stability is provided in terms of matrix inequalities. The problem of global asymptotic stability is investigated for 2D discrete F-M systems with state saturation and time-varying delays in "Stability analysis of state saturation $2 D$ discrete time-delay systems based on F$M$ model" by D. Chen and H. Yu. By constructing a delaydependent 2D discrete Lyapunov functional and introducing a nonnegative scalar based on a row diagonally dominant matrix, a sufficient condition is proposed to guarantee the global asymptotic stability of the addressed systems by solving the linear matrix inequalities (LMIs). In the work entitled "Impulsive vaccination SEIR model with nonlinear incidence rate and time delay" by $\mathrm{D}$. Li et al., a new impulsive vaccination SEIR epidemic model with time delay and nonlinear incidence rate is established. A sufficient condition is given to guarantee the stability of the disease-free periodic solution and the persistence of the model. Subsequently, in the paper entitled "Synchronization of complex dynamical networks with nonidentical nodes and derivative coupling via distributed adaptive control" by $\mathrm{M}$. Shi et al., the distributed adaptive learning laws of periodically time-varying and constant parameters are designed and the distributed adaptive control is constructed.
The design of various controllers has long been the main stream of research topics and much effort has been made for complex systems. In the paper entitled " $H_{\infty}$ control of pairwise distributable large-scale TS fuzzy systems" by A. Filasová and D. Krokavec, the partially decentralized control problems are studied for pairwise distributable large-scale TS fuzzy systems. Sufficient conditions are presented to ensure the stability and $H_{\infty}$ performance. Subsequently, in the work entitled "Stabilization of a class of stochastic nonlinear systems" by N. Noroozi et al., two nonlinear controllers are given and applied to a guidance system in stochastic setting. Firstly, an adaptive control law for the guidance system is presented. Secondly, a robust finite-time control scheme is proposed to stabilize a class of nonlinear stochastic systems such that the closed loop system is stable. A new approach for self-triggered control is proposed in "Selftriggered model predictive control using optimization with prediction horizon one" by K. Kobayashi and K. Hiraishi from the viewpoint of model predictive control (MPC). The difficulty of self-triggered MPC is explained. Accordingly, the one-step input-constrained problem and the $\mathrm{N}$-step input-constrained problem are formulated and solved. In the paper entitled "Robust $H_{\infty}$ control for discrete-time stochastic interval system with time delay" by S. Yu et al., the robust $H_{\infty}$ control problem is investigated for discrete-time stochastic interval system with time delay. By constructing appropriate Lyapunov-Krasovskii functional, sufficient conditions are given to ensure the existence of the robust $H_{\infty}$ controller for addressed systems. By employing the Takagi-Sugeno (TS) fuzzy linearization approach, the linear parameter varying (LPV) gain scheduling control problem is studied in "Statefeedback $H_{\infty}$ control for LPV system using T-S fuzzy linearization approach" by Y. Hu et al. By constructing the piecewise parameter-dependent Lyapunov function, the LPV T-S fuzzy gain scheduling control law is designed. In the paper entitled " $H_{\infty}$ control for two-dimensional Markovian jump systems with state-delays and defective mode information" by Y. Wei et al., the problem of $H_{\infty}$ control is discussed for a class of two-dimensional (2-D) Markovian jump linear systems (MJLSs) with state-delays and defective mode information. An extended model predictive control algorithm is given in "LMI-based model predictive control for a class of constrained uncertain fuzzy Markov jump system" by T. Yang and H. R. Karimi to address the problem of constrained robust model predictive control. By introducing two external parameters, new upper bounds on arbitrarily long time intervals are derived and less conservative results are given. In the work entitled "Active control of oscillation patterns in the presence of multiarmed pitchfork structure of the critical manifold of singularly perturbed system" by R. Vrabel et al., the possibility of control of oscillation patterns is analyzed for nonlinear dynamical systems without the excitation of oscillatory inputs. A general method is developed for the partition of the space of initial states to the areas allowing active control of the stable steady-state oscillations. The implementation of pair-wise decomposition is discussed in "Coordinated control for a group of interconnected pairwise subsystems" by C. $\mathrm{Ma}$ and X.-B. Chen for an interconnected system with uncertainties. The proposed controller scheme is applied on 
a four-area power system to illustrate the effectiveness of the main results.

In the past decades, the issues of filter design and fault detection have received considerable research interests and have found successful applications in a wide range of practical engineering domains. In the paper entitled "Robust $H_{\infty}$ filtering for a class of uncertain Markovian jump systems with time delays" by Y. Yang and J. Lu, the problem of robust $H_{\infty}$ filtering is studied for a class of uncertain timedelay systems with Markovian jumping parameters and norm-bounded time-varying parameter uncertainties. The robust filter is designed in "Finite-frequency filter design for networked control systems with missing measurement" by $\mathrm{D}$. Ye et al. for networked control systems (NCSs) with random missing measurements. A finite-frequency stochastic $H_{\infty}$ performance is given and a sufficient condition is derived to guarantee desired performance. In the work entitled "Intermittent fault detection for uncertain networked systems" by X. He et al., the robust fault detection problem is studied for a class of discrete-time networked systems with multiple state delays and unknown input. Polytopictype parameter uncertainty in the state-space model matrices is considered. A novel measurement model is employed to account for both the random measurement delays and the stochastic data missing (package dropout) phenomenon. By converting the addressed robust fault detection problem into an alternative robust $H_{\infty}$ filtering problem of a certain Markovian jumping system, a sufficient condition for the existence of the desired robust fault detection filter is presented. The work entitled "Adaptive fault detection with two time-varying control limits for nonlinear and multimodal processes" by J. Li et al. is concerned with the two time-varying control limits design used for on-line fault detection for the multimode and nonlinear process. Mahalanobis distances among samples and super ball domains of mean and variance of samples are computed by a just-in-time (JIT) approach to reduce and update the training data set as queries being detected. In the paper entitled "Fault diagnosis for linear discrete systems based on an adaptive observer" by J. Liu et al., a fault diagnosis algorithm is developed to estimate the fault for a class of linear discrete systems based on an adaptive fault estimation observer. An observer gain matrix and adaptive adjusting rule of the fault estimator are designed. The proposed adaptive regulating algorithm can guarantee the negativity of the first order difference of a Lyapunov discrete function so that the observer is ensured to be stable and fault estimation errors are convergent. The problem of robust fault-tolerant tracking control is studied in "Adaptive finite-time control for a flexible hypersonic vehicle with actuator fault" by J. Wang et al. Simulation on the longitudinal model of a flexible air-breathing hypersonic vehicle (FAHV) with actuator faults and uncertainties is conducted. An adaptive fault-tolerant control strategy is presented based on practical finite-time sliding mode method such that the velocity and altitude track their desired commands in finite time with the partial loss of actuator effectiveness. The adaptive update laws are used to estimate the upper bound of uncertainties and the minimum value of actuator efficiency factor.
Over the past decades, the applications of various control schemes have received considerable research interests. In the work entitled "An overview of distributed energy-efficient topology control for wireless adhoc networks" by M. J. Abbasi et al., most recent energy efficient topology control algorithms in WSNs and adhoc network are classified and studied. The topology control aims of the existing algorithms are characterized into three main types: energy efficiency, network capacity, and energy balancing. The most famous and recent topology control algorithms based on their features are reviewed and compared in each goals category. In the paper entitled "Proportional derivative control with inverse deadzone for pendulum systems" by J. Rubio et al., a proportional derivative controller with inverse dead-zone is proposed for the control of pendulum systems with dead-zone inputs. By using the Lyapunov analysis, the asymptotic stability of the proposed technique is guaranteed. Based on the hot rolling process, a load distribution optimization model is established in "Load distribution of evolutionary algorithm for complexprocess optimization based on differential evolutionary strategy in Hot Rolling Process" by X. Yang et al. The rolling force ratio distribution and good strip shape are integrated as two indicators of objective function in the optimization model. The evolutionary algorithm for complex-process optimization (EACOP) is introduced. The paper entitled "Nonlinear robust control of a hypersonic flight vehicle using fuzzy disturbance observer" by L. Zehngdong et al. is concerned with a novel tracking controller design for a hypersonic flight vehicle in complex and volatile environment. The attitude control model is constructed with multivariate uncertainties and external disturbances. The nonlinear disturbance observer is introduced to estimate the influence of uncertainties and disturbances on the flight control system. Also, fuzzy theory is adopted to improve the performance of the nonlinear disturbance observer. By analyzing the topology of China Education and Research Network, it can be concluded that it is different from the common Internet in several aspects as in "Study of evolution model of China education and research network" by G. Mao and N. Zhang. The evolution model of the complex directed network is established which reflects some main characteristics of China Education and Research Network. In the work entitled "On the multipeakon dissipative behavior of the modified coupled Camassa-Holm model for shallow water system" by Z. Shen et al., the dissipative property is taken into account for the modified coupled two-component Camassa-Holm system after wave breaking. Based on the obtained global dissipative solutions to the modified coupled two-component Camassa-Holm system, the dissipative multipeakon solutions are constructed. In the paper entitled "Analysis of the degradation of MOSFETs in switching mode power supply by characterizing source oscillator signals" by X. Zheng et al., the focus is on the detection of incipient faults and on analyzing aging. The wiretap channel with action-dependent states and rate-limited feedback is established in "Wiretap channel with action-dependent states and rate-limited feedback" by X. Yin et al. The capacityequivocation region and secrecy capacity of such a channel are obtained. 


\section{Acknowledgments}

This special issue is a timely reflection of the recent research progress in the area of mathematical control of complex systems. We would like to acknowledge all authors for their efforts in submitting high-quality papers. We are also very grateful to the reviewers for their thorough and on time reviews of the papers.

Zidong Wang

Hamid Reza Karimi

Bo Shen

Jun $\mathrm{Hu}$

Hongli Dong

Xiao $\mathrm{He}$ 


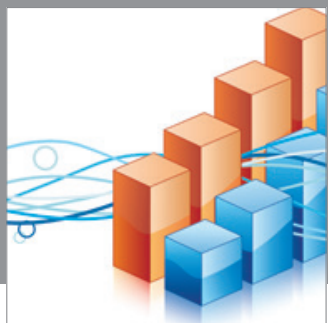

Advances in

Operations Research

mansans

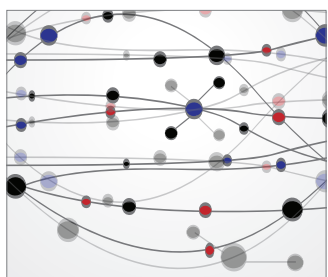

The Scientific World Journal
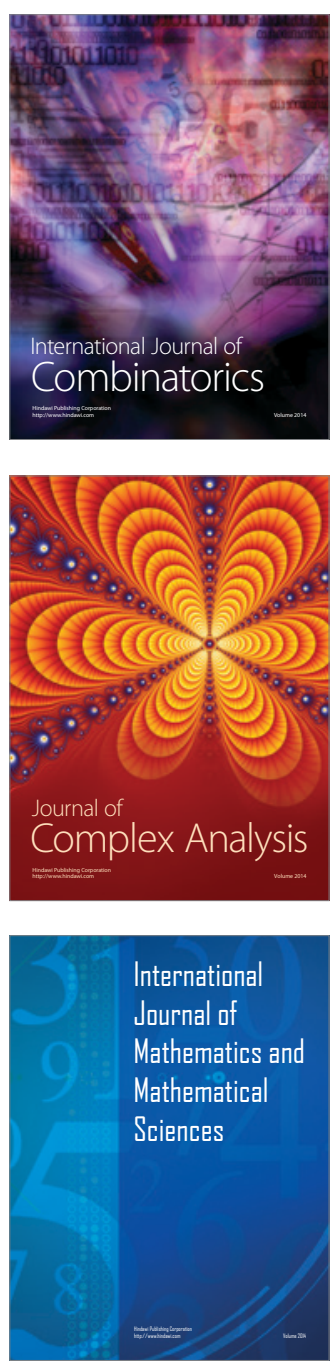
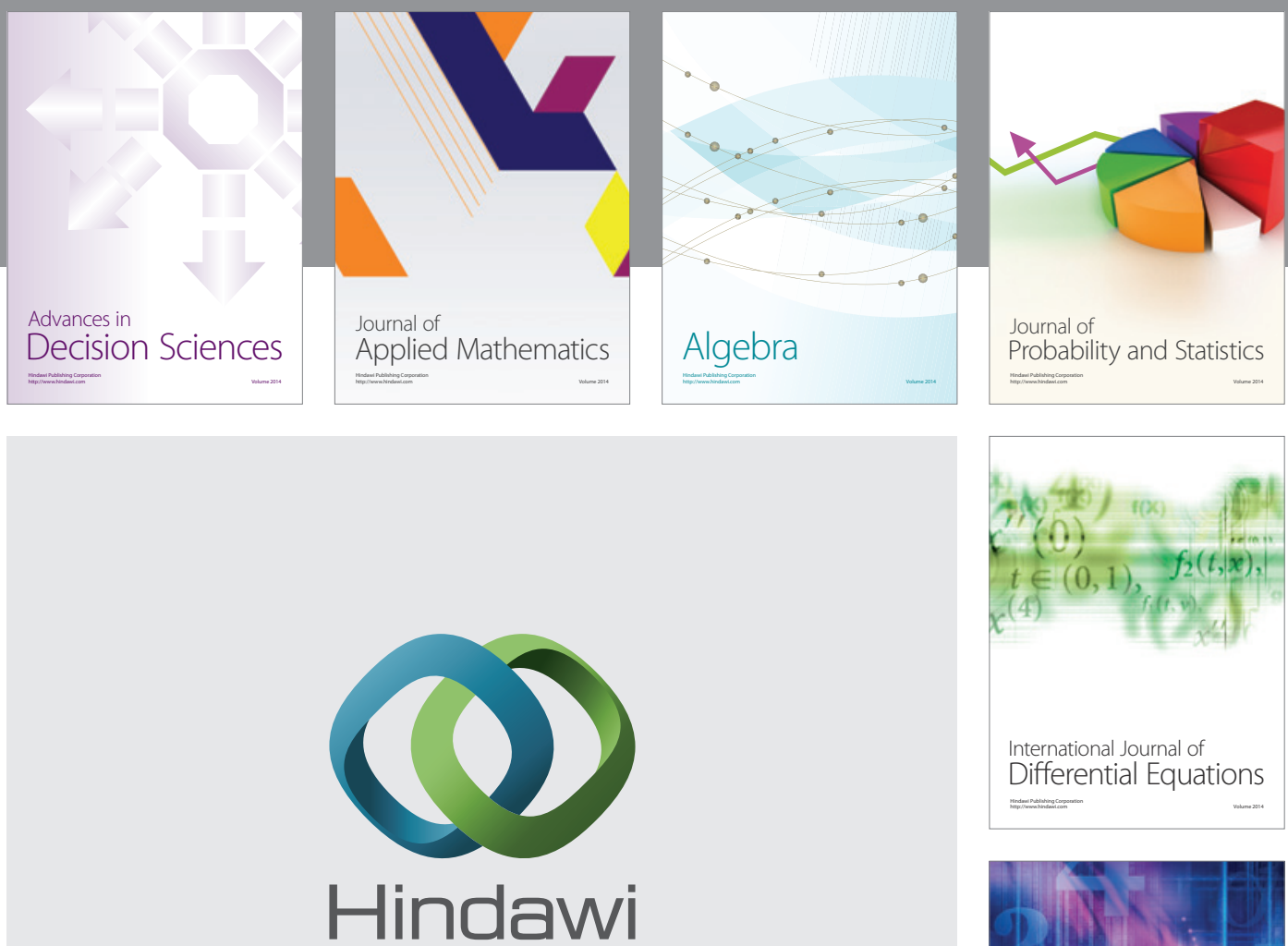

Submit your manuscripts at http://www.hindawi.com
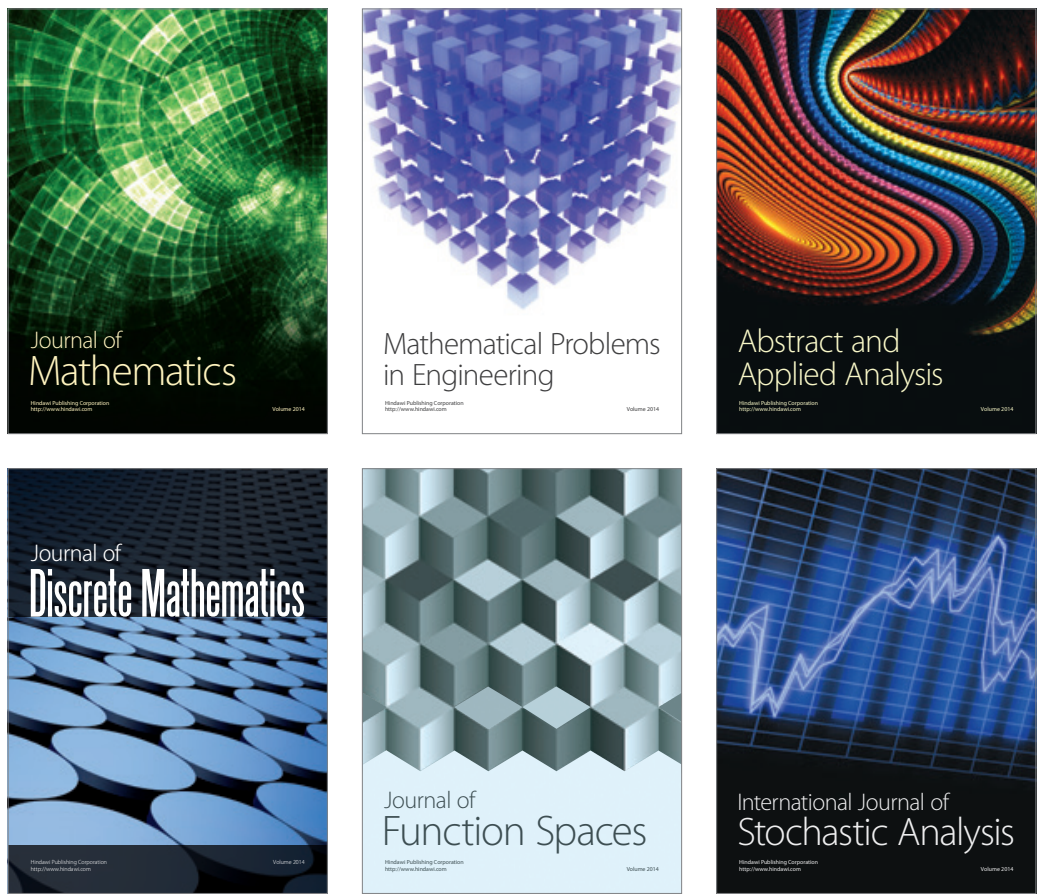

Journal of

Function Spaces

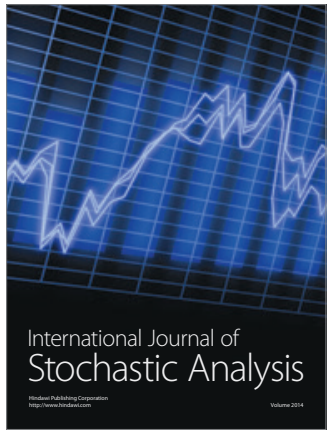

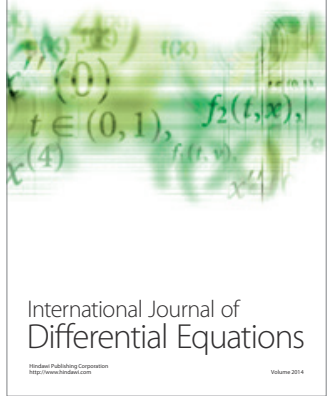
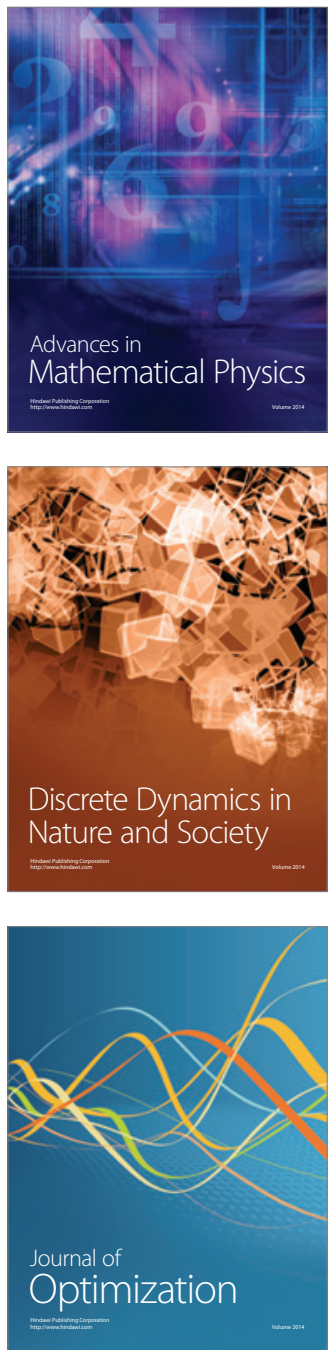